\title{
Prediction of Respiratory Motion with Wavelet-Based Multiscale Autoregression
}

\author{
Floris Ernst, Alexander Schlaefer, and Achim Schweikard \\ Institute of Robotics and Cognitive Systems, University of Lübeck, DE \\ \{ernst, schlaefer, schweikard\}@rob.uni-luebeck.de
}

\begin{abstract}
In robotic radiosurgery, a photon beam source, moved by a robot arm, is used to ablate tumors. The accuracy of the treatment can be improved by predicting respiratory motion to compensate for system delay. We consider a wavelet-based multiscale autoregressive prediction method. The algorithm is extended by introducing a new exponential averaging parameter and the use of the Moore-Penrose pseudo inverse to cope with long-term signal dependencies and system matrix irregularity, respectively. In test cases, this new algorithm outperforms normalized LMS predictors by as much as $50 \%$. With real patient data, we achieve an improvement of around 5 to $10 \%$.
\end{abstract}

\section{Introduction}

To successfully ablate tumors in the body stem using radiosurgery, it is necessary to move the beam source to compensate for the motion of internal organs. This is achieved by recording the motion of the body surface and drawing conclusions about the tumor position [1, 2]. While this method significantly increases the targeting accuracy, the system delay arising from data acquisition and processing and positioning of the treatment beam results in a systematic error. This error can be decreased by predicting the time series of human respiration [3]. We propose to use the wavelet-based multiscale autoregression prediction method introduced in [4] and extend it by introducing an averaging parameter to capture long-term signal dependencies.

\section{Prediction Algorithms}

Let $y$ be the signal we want to predict. Since the respiration time series can be approximated by sinusoidal models, we assume an autoregressive (AR) property:

Definition 1. Let $y$ be a uniformly sampled signal. We say that $y$ is an $A R(M)$ process if there is an integer $M$ such that, when the last $M$ values $y_{n-M+1}, \ldots, y_{n}$ of the signal $y$ are known, for each positive integer $k$ there are weights $w=$ $\left(w_{1}, \ldots, w_{M}\right)^{\mathrm{T}}$ such that for all $m \geq n+k, y_{m}=w^{\mathrm{T}} u_{m-k}$. Here, $u_{m-k}=$ $\left(y_{m-k-M+1}, \ldots, y_{m-k}\right)^{\mathrm{T}}$. 
If breathing motion was perfectly sinusoidal and the values of the weights were known, it would be possible to exactly predict the time series. Since real data is not perfect, we cannot expect to obtain perfect prediction. At best, we can model the current, underlying sinusoidals and automatically adapt the weights to compensate for changes in breathing behavior.

\subsection{The LMS Algorithm}

One way of learning the weights of an $\operatorname{AR}(M)$-process is the LMS algorithm [5], which has been used for a long time in time series prediction and signal analysis.

$$
\begin{aligned}
\hat{y}_{n+k}^{\mathrm{LMS}} & =w_{n}^{\mathrm{T}} u_{n}, \quad w_{n+1}=w_{n}+\mu\left(\hat{y}_{n}^{\mathrm{LMS}}-y_{n}\right) u_{n}, \quad n \geq k, \\
u_{n} & =\left(u_{n, 1}, \ldots, u_{n, M}\right)^{\mathrm{T}}=\left(y_{n-M+1}, \ldots, y_{n}\right)^{\mathrm{T}}, \\
\hat{y}_{1}^{\mathrm{LMS}} & =\cdots=\hat{y}_{k}^{\mathrm{LMS}}=y_{1}, \quad w_{1}=(0, \ldots, 0,1)^{\mathrm{T}}
\end{aligned}
$$

$y_{n}$ is the measured signal at step $n, w_{n}$ the corresponding weight vector and $u_{n}$ is the signal history used in step $n$ to compute $\hat{y}_{n+k}$, the prediction for step $n+k$. For stationary signals, the LMS algorithm is known to perfectly adapt to the system if the learning parameter $\mu$ is chosen properly. Difficulties arise from the fact that in the update term for the weight vector $w$ large signal values lead to a larger correction term, i.e. when provided with two differently scaled versions of a signal, the algorithm produces different results. This problem is somewhat alleviated by using a normalized LMS algorithm (nLMS).

\subsection{The nLMS Algorithms}

To improve the convergence properties of the LMS algorithm (i.e. to make it independent from scaling and increase the rate of convergence), normalized LMS algorithms are used (see [6]).

$$
\begin{aligned}
\hat{y}_{n+k}^{\mathrm{nLMS}_{p}} & =w_{p, n}^{\mathrm{T}} u_{n}, \quad u_{n}=\left(u_{n, 1}, \ldots, u_{n, M}\right)^{\mathrm{T}}=\left(y_{n-M+1}, \ldots, y_{n}\right)^{\mathrm{T}}, \\
w_{p, n+1} & =w_{p, n}+\mu\left(\hat{y}_{n}^{\mathrm{nLMS}}-y_{n}\right) f_{p, n}, \\
\hat{y}_{1}^{\mathrm{nLMS}_{p}} & =\cdots=\hat{y}_{k}^{\mathrm{nLMS}_{p}}=y_{1}, \quad w_{p, 1}=(0, \ldots, 0,1)^{\mathrm{T}}
\end{aligned}
$$

Here, the error correction term $f_{p, n}$ is defined as follows:

$$
\left(f_{p, n}\right)_{i}=\frac{\left|u_{n, i}\right|^{p-1} \operatorname{sgn}\left(u_{n, i}\right)}{\left\|u_{n}\right\|_{p}^{p}}, \quad\left(f_{\infty, n}\right)_{i}=\frac{\delta_{i, l}}{u_{n, l}}, \quad l=\underset{j=1, \ldots, M}{\operatorname{maxind}}\left|u_{n, j}\right|
$$

In our case, we only considered the special case of $p=2$ (i.e. normalization with respect to the Euclidean norm), whence the algorithm reduces to the following:

$$
\begin{aligned}
& \hat{y}_{n+k}^{\mathrm{nLMS}_{2}}=w_{2, n}^{\mathrm{T}} u_{n} \\
& w_{2, n+1}=w_{2, n}+\mu\left(\hat{y}_{n}^{\mathrm{nLMS} 2}-y_{n}\right) f_{2, n}=w_{2, n}+\mu\left(\hat{y}_{n}^{\mathrm{nLMS}_{2}}-y_{n}\right) \frac{u_{n}}{\alpha+\left\|u_{n}\right\|_{2}^{2}}
\end{aligned}
$$

To avoid division by zero, a small parameter $\alpha$ (typically $10^{-4}$ ) is introduced in the denominator of the error term $f_{2, n}$ (Equation 4). Further improvements are possible by preprocessing the signal. This is done with the wavelet based LMS algorithm. 


\subsection{The wLMS Algorithm}

Renaud, Starck and Murtagh in 4] propose to predict an autoregressive signal using its á trous wavelet decomposition. It is computed in a sequential way (Equation 5); therefore, on-line processing of the signal is possible.

$$
c_{0, n}=y_{n}, \quad c_{j+1, n}=\frac{1}{2}\left(c_{j, n-2^{j}}+c_{j, n}\right), \quad W_{j+1, n}=c_{j, n}-c_{j+1, n}
$$

Computing this decomposition up level $J$, we get $J+1$ bands representing the signal: $W_{1}, \ldots, W_{J}$, the wavelet scales, and $c_{J}$, the smoothed signal, such that $y_{n}=W_{1, n}+\cdots+W_{J, n}+c_{J, n}$. Hence, it is possible to separately predict the individual bands. This is done by selecting regression depths $a_{j}$ for each level $W_{j}$ and $a_{J+1}$ for the band $c_{J}$. Assuming the $\operatorname{AR}\left(2^{a_{j}}\right)$-property on the individual bands and knowledge of the weight vectors $w_{j}$, we get the multiscale autoregression (MAR) forecasting formula:

$$
\begin{aligned}
\hat{y}_{n+k}^{\mathrm{MAR}} & =\sum_{j=1}^{J} w_{j}^{\mathrm{T}} \tilde{W}_{n, j}+w_{J+1}^{\mathrm{T}} \tilde{c}_{n} \\
\tilde{W}_{n, j} & =\left(W_{j, n-2^{j} \cdot 0}, W_{j, n-2^{j} \cdot 1}, \ldots, W_{j, n-2^{j}\left(a_{j}-1\right)}\right)^{\mathrm{T}} \\
\tilde{c}_{n} & =\left(c_{J, n-2^{J} \cdot 0}, c_{J, n-2^{J} \cdot 1}, \ldots, c_{J, n-2^{J}\left(a_{J+1}-1\right)}\right)^{\mathrm{T}}
\end{aligned}
$$

Here, the vectors $\tilde{W}_{n, j}$ and $\tilde{c}_{n, j}$ take the role of the vector $u_{n}$ (the signal history). However, the correct values for the weight vectors $w_{j}$ are not known and need to be adaptively learned. This is done by least mean squares fitting to the last $M$ signal steps, completing the description of the wLMS algorithm:

$$
\begin{aligned}
& B=\left(l_{n-k}, \ldots, l_{n-k-M+1}\right)^{\mathrm{T}}, \quad l_{t}=\left(\tilde{W}_{t, 1}^{\mathrm{T}}, \ldots, \tilde{W}_{t, J}^{\mathrm{T}}, \tilde{c}_{t}^{\mathrm{T}}\right)^{\mathrm{T}} \\
& w=\left(w_{1}^{\mathrm{T}}, \ldots, w_{J+1}^{\mathrm{T}}\right)^{\mathrm{T}}, \quad s_{n}=\left(y_{n}, \ldots, y_{n-M+1}\right)^{\mathrm{T}} \\
& \text { solve } B w=s_{n} \text { (by normal equation) to update } w
\end{aligned}
$$

We extend this approach: Since the matrix of the normal equation used in (7) might not be regular, we have improved the algorithm to cope with irregularity. This is done by replacing $\left(B^{\mathrm{T}} B\right)^{-1} B^{\mathrm{T}}$ by the Moore-Penrose pseudo inverse of $B$. This does not alter the results whenever the rank of $B$ is maximal, it only improves numerical stability. Obviously, for each point in time there is a maximum number of past observations which influence the prediction. However, it is possible that information not contained in the signal history (the vectors $\tilde{W}_{n, j}$ and $\tilde{c}_{n, j}$ ) still influences the future of the time series. To include this information in the prediction, we introduce an exponential averaging parameter $\mu$ and modify the method as follows:

$$
\begin{aligned}
\hat{y}_{n+k}^{\mathrm{wLMS}} & =\sum_{j=1}^{J} w_{n, j}^{\mathrm{T}} \tilde{W}_{n, j}+w_{J+1}^{\mathrm{T}} \tilde{c} \\
w_{n} & =\left(w_{n, 1}^{\mathrm{T}}, \ldots, w_{n, J+1}^{\mathrm{T}}\right), \quad w_{1}=\cdots=w_{k+M-1}=(0, \ldots, 0)^{\mathrm{T}} \\
w_{n+1} & =(1-\mu) w_{n}+\mu B^{+} s_{n}, \quad \mu \in[0,1], \quad n \geq k+M
\end{aligned}
$$


Here, the symbol + denotes the Moore-Penrose pseudo inverse of a matrix. Small values of $\mu$ correspond to a prediction with high confidence in the past while large values correspond to high confidence in recent observations. Note that with the introduction of $\mu$ there is no explicit signal history length and that setting $\mu=1$ will yield the results of the original MAR algorithm.

\section{$3 \quad$ Experiments}

The algorithms presented above were applied to several simulated and real respiratory signals to compare their performance. For prediction tests, the algorithms were allowed to stabilize on the first 2,000 sampling points and the prediction result was evaluated on the remaining data, both in absolute numbers $[\mu \mathrm{m}]$ and as a percentage of the nRMS error of the delayed curve.

\subsection{Simulated Data}

Sinusoidal signals were used to simulate breathing data. The signals are shown in Figure 1 Part (e) was generated from two breathing cycles recorded during a

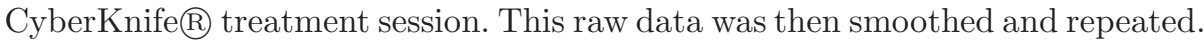
All signals are sampled at $40 \mathrm{~Hz}$ and have a length of 10,000 sampling points.

In a second step, to simulate real-world data, the signals from Figure 1 were corrupted by additive Gaussian noise of four different amplitudes (with standard deviations of $0.05,0.10,0.20$ and $0.50 \mathrm{~mm}$ ).

For the wLMS algorithm, $M$ was set to 2 and $J$ was set to 7 . The values of the $a_{j}$ were automatically determined: the á trous wavelet decomposition of the first 2,000 points was computed and then the coefficients were set according to Equation 9.

$$
a_{j}=15\left\lceil\frac{W_{j}^{\mathrm{T}} W_{j}}{\left(y-c_{J}\right)^{\mathrm{T}}\left(y-c_{J}\right)}\right\rceil, \quad a_{J+1}=2
$$

In case of the uncorrupted signals, for both the LMS and the $\mathrm{nLMS}_{2}$ algorithms, $M$ was set to 6 ; in case of the noisy signals, $M$ was set to 8 . Furthermore, to take advantage of the noise filtering ability of the wLMS algorithm, the coefficients
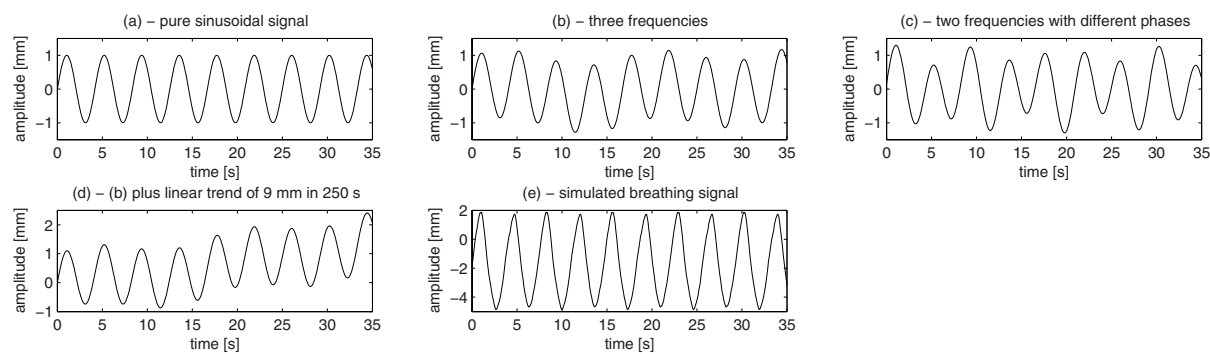

Fig. 1. Simulated data 
Table 1. nRMS errors on simulated data (optimal values are in bold print)

\begin{tabular}{|c|c|r|r|r|r|r|r|r|r|r|}
\hline \multirow{2}{*}{ sig. } & \multirow{2}{*}{ delay } & \multicolumn{3}{|c|}{ LMS } & \multicolumn{3}{|c|}{ nLMS } & \multicolumn{3}{|c|}{ wLMS } \\
& abs. & rel. & $\mu$ & abs. & rel. & $\mu$ & abs. $[\mu \mathbf{m}]$ & rel. [\%] & $\mu$ \\
\hline \multirow{4}{*}{ (a) } & 5 & 0.04 & 0.03 & 0.0872 & $\mathbf{0}$ & 0 & 0.1472 & $\mathbf{0}$ & 0 & 0.0632 \\
& 10 & 81.84 & 30.88 & 0.0424 & $\mathbf{0}$ & 0 & 0.1152 & $\mathbf{0}$ & 0 & 0.0272 \\
& 15 & 26.05 & 6.60 & 0.0260 & 0.00 & 0.00 & 0.0960 & $\mathbf{0}$ & 0 & 0.0552 \\
& 20 & 0.00 & 0.00 & 0.0340 & $\mathbf{0}$ & 0 & 0.0592 & $\mathbf{0}$ & 0 & 0.0264 \\
\hline & 5 & 28.05 & 21.05 & 0.0400 & 4.05 & 3.04 & 0.0536 & $\mathbf{2 . 9 2}$ & 2.19 & 1.0000 \\
(b) & 10 & 114.80 & 43.26 & 0.0184 & 15.42 & 5.81 & 0.0336 & $\mathbf{1 0 . 4 7}$ & 3.94 & 1.0000 \\
& 15 & 216.32 & 54.75 & 0.0128 & 36.43 & 9.22 & 0.0248 & $\mathbf{2 2 . 2 9}$ & 5.64 & 1.0000 \\
& 20 & 304.25 & 58.36 & 0.0124 & 67.61 & 12.97 & 0.0172 & $\mathbf{3 8 . 1 2}$ & 7.31 & 1.0000 \\
\hline & 5 & 29.42 & 21.79 & 0.0388 & 3.95 & 2.92 & 0.0488 & $\mathbf{2 . 8 7}$ & 2.12 & 1.0000 \\
(c) & 10 & 65.66 & 24.43 & 0.0416 & 14.33 & 5.33 & 0.0272 & $\mathbf{1 0 . 4 6}$ & 3.89 & 1.0000 \\
& 15 & 232.36 & 58.06 & 0.0108 & 32.28 & 8.06 & 0.0216 & $\mathbf{2 2 . 7 0}$ & 5.67 & 1.0000 \\
& 20 & 301.93 & 57.16 & 0.0132 & 57.45 & 10.87 & 0.0192 & $\mathbf{3 9 . 4 4}$ & 7.47 & 1.0000 \\
\hline & 5 & 92.98 & 69.73 & 0.0026 & 29.61 & 22.21 & 0.1344 & $\mathbf{1 3 . 9 6}$ & 10.47 & 1.0000 \\
(d) & 10 & 230.80 & 86.93 & 0.0012 & 147.41 & 55.52 & 0.0632 & $\mathbf{5 1 . 5 2}$ & 19.40 & 1.0000 \\
& 15 & 395.33 & 100.00 & 0.0000 & 380.35 & 96.21 & 0.0056 & $\mathbf{1 1 2 . 4 2}$ & 28.44 & 1.0000 \\
& 20 & 521.65 & 100.00 & 0.0000 & 520.79 & 99.84 & 0.0002 & $\mathbf{1 9 5 . 4 2}$ & 37.46 & 1.0000 \\
\hline & 5 & 148.84 & 30.52 & 0.0038 & 121.96 & 25.01 & 0.0160 & $\mathbf{9 9 . 9 0}$ & 20.48 & 0.4360 \\
(e) & 10 & 421.10 & 43.70 & 0.0016 & 255.05 & 26.47 & 0.0136 & $\mathbf{2 1 3 . 7 6}$ & 22.18 & 0.3472 \\
& 15 & 687.55 & 48.24 & 0.0012 & 395.08 & 27.72 & 0.0120 & $\mathbf{3 3 6 . 8 7}$ & 23.63 & 0.3128 \\
& 20 & 919.61 & 49.22 & 0.0012 & 550.66 & 29.48 & 0.0108 & $\mathbf{2 5 8 . 2 5}$ & 13.82 & 0.0024 \\
\hline
\end{tabular}

$a_{1}$ and $a_{2}$ were, in this case, chosen to be zero. The predictors were tested with $\mu$ from 0 to 1 (in steps of 0.0001 ) and the best result was selected.

The absolute $[\mu \mathrm{m}]$ and relative [\%] nRMS errors on the signals without noise are shown in Table 1. The computations with a prediction horizon of 5 time steps were repeated on the signals corrupted by noise. The predicted curves were compared to the uncorrupted signals. Noise amplitude at level 1 is lower than at level 2 and so on (see Table 2). Thirdly, the computations for the wLMS algorithm were repeated after setting coefficients $a_{1}$ through $a_{4}$ to zero (See Table 3). In all but four cases the wLMS algorithm was able to outperform the $\mathrm{nLMS}_{2}$ algorithm by up to $50 \%$. As a drawback, we see that discarding the first four bands of the wavelet coefficients can lead to a loss of information, since high-frequency parts of the real signal can be present in these bands. This can be seen in Table 3: the nRMS value for noise level 1 of signal (e), in italics, has increased in comparison to the value in Table 2 .

\subsection{Real Data}

The algorithms were also tested on breathing signals of three patients treated with the CyberKnifeß system. The signals all have a length of 30,000 sampling points at a rate of approximately $26 \mathrm{~Hz}$, Figure 2. In this test, a grid search for the optimal values of $M$ and $\mu$ was performed; the prediction horizon was set to five time steps. The wLMS coefficients $a$ were selected according to Equation9. The results 
Table 2. nRMS errors on simulated data, corrupted by noise, at a delay of 5

\begin{tabular}{|c|c|c|c|c|c|c|c|c|c|c|}
\hline \multirow{2}{*}{ sig. } & \multirow{2}{*}{$\begin{array}{l}\text { noise } \\
\text { level }\end{array}$} & \multicolumn{3}{|c|}{ LMS } & \multicolumn{3}{|c|}{$\overline{\mathbf{n L M S}_{2}}$} & \multicolumn{3}{|c|}{ wLMS } \\
\hline & & abs. & rel. & $\mu$ & abs. & rel. & $\mu$ & abs. & rel. & $\mu$ \\
\hline & 1 & 83.02 & 58.30 & 0.0088 & 74.77 & 52.51 & 0.0248 & 34.26 & 24.06 & 0.004 \\
\hline & 2 & 150.16 & 90.02 & 0.0020 & 131.07 & 78.58 & 0.0168 & 97.82 & 58.64 & 0.0032 \\
\hline & 3 & 240.66 & 100.00 & 0.0000 & 199.88 & 83.06 & 0.0136 & 194.31 & 80.74 & 0.0032 \\
\hline & 4 & 519.40 & 100.00 & 0.0000 & 279.70 & 53.85 & 0.0112 & 348.74 & 67.14 & 0.0016 \\
\hline & 1 & 3.26 & 8.40 & 0.0084 & 75.87 & $\overline{53.22}$ & 0.0 & 51.03 & 35.79 & 0.1 \\
\hline & 2 & 150.19 & 89.96 & 0.0020 & 131.40 & 78.70 & 0.0152 & $|116.67|$ & 69.89 & 0.1144 \\
\hline & 3 & 240.60 & 100.00 & 0.0000 & 198.04 & 82.31 & 0.0128 & 234.91 & 97.63 & 0.1168 \\
\hline & 4 & 519.33 & 00.00 & 0.0000 & 280.77 & 54.06 & 0.0104 & 389.95 & 75.09 & 0.0016 \\
\hline & 1 & 83.41 & 57.90 & 0.0084 & 76.06 & 52.80 & 0.0240 & 50.69 & 35.19 & 0.1096 \\
\hline & 2 & 150.45 & 89.43 & 0.0020 & 131.29 & 78.04 & 0.0176 & 116.11 & 69.01 & 0.1136 \\
\hline & 3 & 241.37 & 100.00 & 0.0000 & 200.16 & 82.93 & 0.0112 & 236.41 & 97.94 & 0.1200 \\
\hline & 4 & 520.43 & 100.00 & 0.0000 & 282.72 & 54.32 & 0.0104 & 436.60 & 83.89 & 0.0896 \\
\hline \multirow{4}{*}{ (d) } & 1 & 113.00 & 79.22 & 0.0012 & 75.50 & 52.93 & 0.1112 & 80.65 & 56.54 & 0.2024 \\
\hline & 2 & 147.05 & 88.054 & 0.0012 & 116.91 & 70.00 & 0.1024 & 139.32 & 83.42 & 0.1736 \\
\hline & 3 & 240.67 & 100.00 & 0.0000 & 179.44 & 74.56 & 0.0880 & 258.48 & 107.40 & 0.1648 \\
\hline & 4 & 519.40 & 100.00 & 0.0000 & 314.46 & 60.54 & 0.0536 & 456.40 & 87.87 & 0.1208 \\
\hline \multirow{4}{*}{$(\epsilon$} & 1 & 161.13 & 32.85 & 0.0016 & 147.10 & 29.99 & 0.0112 & 129.46 & 26.39 & 0.212 \\
\hline & 2 & 208.63 & 42.03 & 0.0012 & 194.32 & 39.14 & 0.0104 & 157.95 & 31.82 & 0.1472 \\
\hline & 3 & 340.06 & 64.6 & & 301.66 & 57.34 & 0.0 & 230.39 & 43.79 & 0.1088 \\
\hline & 4 & 707.60 & 100.00 & 0.0000 & 590.65 & 83.47 & 0.0120 & 503.16 & 71.11 & 0.0024 \\
\hline
\end{tabular}

Table 3. wLMS prediction with coefficients $a_{1}$ through $a_{4}$ set to 0

\begin{tabular}{|c|c|r|r|c|}
\hline \multirow{2}{*}{ signal } & noise & \multicolumn{3}{|c|}{ wLMS } \\
& level & abs. & rel. & $\mu$ \\
\hline \multirow{4}{*}{ (a) } & 1 & $\mathbf{5 . 8 6}$ & 4.12 & 0.0040 \\
& 2 & $\mathbf{1 8 . 9 6}$ & 11.37 & 0.0032 \\
& 3 & $\mathbf{5 6 . 9 4}$ & 23.66 & 0.0024 \\
& 4 & $\mathbf{1 7 2 . 1 2}$ & 33.14 & 0.0016 \\
\hline \multirow{4}{*}{ (b) } & 1 & $\mathbf{4 1 . 9 3}$ & 29.41 & 0.1000 \\
& 2 & $\mathbf{6 6 . 0 8}$ & 39.58 & 0.0744 \\
& 3 & $\mathbf{1 2 3 . 0 8}$ & 51.16 & 0.0552 \\
& 4 & $\mathbf{2 3 7 . 3 4}$ & 45.70 & 0.0016 \\
\hline \multirow{4}{*}{ (c) } & 1 & $\mathbf{4 1 . 3 5}$ & 28.70 & 0.0976 \\
& 2 & $\mathbf{6 5 . 6 0}$ & 38.99 & 0.0728 \\
& 3 & $\mathbf{1 2 1 . 9 9}$ & 50.54 & 0.0552 \\
& 4 & 306.53 & 58.90 & 0.0016 \\
\hline
\end{tabular}

\begin{tabular}{|c|c|r|r|c|}
\hline \multirow{2}{*}{ signal } & noise & \multicolumn{3}{|c|}{ wLMS } \\
& level & abs. & rel. & $\mu$ \\
\hline \multirow{4}{*}{ (d) } & 1 & 77.85 & 54.58 & 0.1896 \\
& 2 & $\mathbf{1 1 6 . 1 1}$ & 69.53 & 0.1448 \\
& 3 & 182.24 & 75.72 & 0.1120 \\
& 4 & 418.66 & 80.60 & 0.0920 \\
\hline \multirow{4}{*}{$(\mathrm{e})$} & 1 & 130.23 & 26.55 & 0.2152 \\
& 2 & $\mathbf{1 5 6 . 5 2}$ & 31.53 & 0.1432 \\
& 3 & $\mathbf{2 0 0 . 5 7}$ & 38.13 & 0.0896 \\
& 4 & $\mathbf{2 7 9 . 7 8}$ & 39.54 & 0.0032 \\
\hline
\end{tabular}

obtained are shown in Table 4 Furthermore, a certain amount of smoothing is permitted, since the real data is inevitably corrupted by measurement noise. Hence, we repeated our computation of the prediction obtained using the wLMS algorithm for two cases: coefficients $a_{1}$ and $a_{2}$ set to zero and coefficients $a_{1}$ through $a_{4}$ set to zero. These results are shown in Table 5. As with the simulated data, the wLMS algorithm again outperforms the $\mathrm{nLMS}_{2}$ algorithm which, 

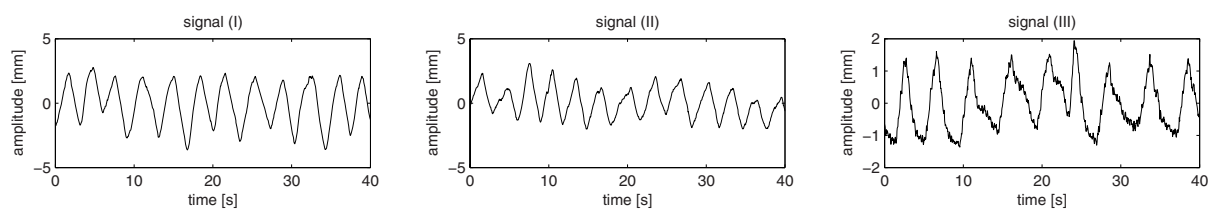

Fig. 2. Real data of three different, rather difficult, breathing signals

Table 4. nRMS errors on real data at a prediction horizon of 5

\begin{tabular}{|c|c|c|c|c|c|c|c|c|c|c|c|c|}
\hline \multirow{2}{*}{ sig. } & \multicolumn{4}{|c|}{ LMS } & \multicolumn{4}{|c|}{ nLMS $_{2}$} & \multicolumn{4}{|c|}{ wLMS } \\
\hline & abs. & rel. & $\mu$ & M & abs. & rel. & $\mu$ & $\mathrm{M}$ & abs. & rel. & $\mu$ & M \\
\hline (I) & 293.56 & 52.33 & 0.0010 & 5 & 260.64 & 46.47 & 0.0064 & 4 & 237.11 & 42.27 & 0.6184 & 2 \\
\hline (II & 245.77 & 62.36 & 0.0014 & 5 & 230.82 & 58.57 & 0.0032 & 4 & 216.18 & 54.85 & 0.5456 & 2 \\
\hline (III) & 415.98 & 70.22 & 0.0001 & 17 & 398.97 & 67.35 & 0.0032 & 9 & 383.95 & 64.81 & .3544 & 2 \\
\hline
\end{tabular}

Table 5. wLMS on real data. $a_{1}$ and $a_{2}$ set to 0 (left); $a_{1}$ through $a_{4}$ set to 0 (right).

\begin{tabular}{|c|c|c|c|c|}
\hline sig. & \multicolumn{3}{|c|}{ wLMS } \\
& abs. & rel. & $\mu$ & $\mathbf{M}$ \\
\hline (I) & 237.87 & 42.41 & 0.6112 & 2 \\
(II) & 218.52 & 55.45 & 0.5248 & 2 \\
(III) & 389.32 & 65.72 & 0.3208 & 2 \\
\hline
\end{tabular}

\begin{tabular}{|c|c|c|c|c|}
\hline sig. & \multicolumn{4}{|c|}{ wLMS } \\
& abs. & rel. & $\mu$ & M \\
\hline (I) & 241.22 & 43.00 & 0.6080 & 2 \\
(II) & 221.01 & 56.08 & 0.5208 & 2 \\
(III) & 391.00 & 66.00 & 0.3200 & 2 \\
\hline
\end{tabular}

in turn, yields better results than the LMS algorithm. It also becomes clear that this improvement is not as pronounced as for the simulated signals: it lies around 5 to $10 \%$. Furthermore, the smoothing does not lead to further improvement. Unfortunately, we do not know the signal uncorrupted by measurement noise and thus can only compare the predicted signal to the noisy signal. As a result, there is hardly any change in the nRMS error. The predicted signal, however, becomes much smoother and hence is more suitable for determining correlation between chest and tumor motion. Summarizing, smoothing does not noticeably degrade and actually improves the results, whence using it in prediction seems reasonable.

Although the improvement was not huge when working with real world data, it is now clear that selecting the correct parameters for LMS-based algorithms is crucial. To underline this, consider Figure 3. Here, the relative nRMS error of the three algorithms are shown as a function of $\mu$ and $M$. Obviously, selecting $\mu$ and $M$ for the LMS and the $\mathrm{nLMS}_{2}$ algorithms is very difficult: a slightly off-optimum value can lead to complete breakdown of the prediction. On the other hand, working with the wLMS algorithm is by far easier. All our results show that the optimal value for $M$ is 2 - for both simulated and real signals. 

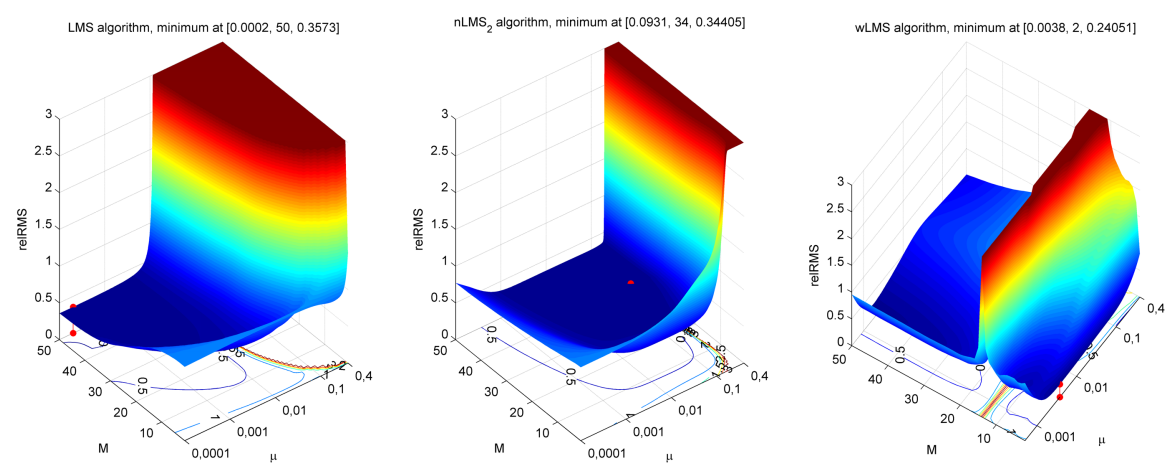

Fig. 3. Relative nRMS error of the LMS (left), nLMS 2 (center) and wLMS algorithms on signal (a) at noise level 1 with a prediction horizon of 5 (logarithmic scale in $\mu$ )

\section{Conclusions}

The presented extension of the wavelet-based MAR algorithm leads to improved results compared with the LMS and the $\mathrm{nLMS}_{2}$ algorithms for both simulated and real signals. For real data, the improvement is less pronounced. However, we have shown that, for the wLMS algorithm, selecting the correct parameters is by far easier. Therefore, the use of the wLMS algorithm in prediction of human respiration is a viable alternative to the $\mathrm{nLMS}_{2}$ algorithm.

\section{References}

1. Schweikard, A., Glosser, G., Bodduluri, M., Adler, J.: Robotic motion compensation for respiratory motion during radiosurgery. Journal of Computer-Aided Surgery 5(4), 263-277 (2000)

2. Schweikard, A., Shiomi, H., Adler, J.: Respiration tracking in radiosurgery. Medical Physics 31(10), 2738-2741 (2004)

3. Sharp, G.C., Jiang, S.B., Shimizu, S., Shirato, H.: Prediction of respiratory tumour motion for real-time image-guided radiotherapy. Physics in Medicine and Biology 49, 425-440 (2004)

4. Renaud, O., Starck, J.L., Murtagh, F.: Prediction based on a multiscale decomposition. International Journal of Wavelets, Multiresolution and Information Processing 1(2), 217-232 (2003)

5. Haykin, S.: Adaptive Filter Theory, 4th edn. Prentice Hall, Englewood Cliffs, NJ (2002)

6. Douglas, S.C.: A family of normalized LMS algorithms. IEEE Signal Processing Letters 1(3), 49-55 (1994) 